\title{
The Formation of Hexagonal-shaped InGaN-nanodisk on GaN- Nanowire Observed in Plasma Source Molecular Beam Epitaxy
}

Tien Khee $\mathrm{Ng}^{\mathrm{a}}$, Anwar Gasim ${ }^{\mathrm{a}}$, Dongkyu Cha ${ }^{\mathrm{b}}$, Bilal Janjua ${ }^{\mathrm{a}}$, Yang Yang ${ }^{\mathrm{b}}$, Shafat Jahangir ${ }^{\mathrm{c}}$, Chao Zhao ${ }^{\mathrm{a}, \mathrm{b}}$, Pallab Bhattacharya ${ }^{\mathrm{c}}$, and Boon Siew Ooi ${ }^{* a}$

${ }^{a}$ Photonics Laboratory, King Abdullah University of Science and Technology (KAUST), Thuwal 21534, Saudi Arabia.

${ }^{\mathrm{b}}$ Imaging and Characterization Core Laboratory, King Abdullah University of Science and Technology (KAUST), Thuwal 21534, Saudi Arabia.

${ }^{\mathrm{c}}$ Department of Electrical Engineering and Computer Science, University of Michigan, 1301, Beal Avenue, Ann Arbor, Michigan 48109-2122, USA.

\begin{abstract}
We report on the properties and growth kinetics of defect-free, photoluminescence (PL) efficient mushroom-like nanowires (MNWs) in the form of $\sim 30 \mathrm{~nm}$ thick hexagonal-shaped InGaN-nanodisk on GaN nanowires, coexisting with the conventional rod-like InGaN-on-GaN nanowires (RNWs) on (111)-silicon-substrate. When characterized using confocal microscopy (CFM) with $458 \mathrm{~nm}$ laser excitation, while measuring spontaneous-emission at fixed detection wavelengths, the spatial intensity map evolved from having uniform pixelated emission, to having only an emission ring, and then a round emission spot. This corresponds to the PL emission with increasing indium composition; starting from emission mainly from the RNW, and then the $540 \mathrm{~nm}$ emission from one MNWs ensemble, followed by the $590 \mathrm{~nm}$ emission from a different MNW ensemble, respectively. These hexagonal-shaped InGaN-nano-disks ensembles were obtained during molecular-beam-epitaxy (MBE) growth. On the other hand, the regular rod-like InGaN-on-GaN nanowires (RNWs) were emitting at a shorter peak wavelength of $490 \mathrm{~nm}$. While the formation of InGaN rod-like nanowire is well-understood, the formation of the hexagonal-shaped InGaN-nanodisk-on-GaN-nanowire requires further investigation. It was postulated to arise from the highly sensitive growth kinetics during plasma-assisted $\mathrm{MBE}$ of InGaN at low temperature, i.e. when the substrate temperature was reduced from $800{ }^{\circ} \mathrm{C}$ (GaN growth) to $<600{ }^{\circ} \mathrm{C}$ (InGaN growth), during which sparsely populated metal-droplet formation prevails and further accumulated more indium adatoms due to a higher cohesive bond between metallic molecules.
\end{abstract}

Keywords: InGaN / GaN, Nanowires, Catalyst-free Growth Kinetics, Nitrogen-plasma Source Molecular Beam Epitaxy, Confocal Microscopy, Photoluminescence Spectroscopy, Scanning Electron Microscopy

\section{INTRODUCTION}

Anisotropic epitaxial growth of free-standing GaN [1, 2] and InGaN nanowires [3, 4] presents a key technological advancement in material structure for light-emitter technology. The catalyst-free [5-7] and spontaneous growth of nitrogen-polar GaN nanowires [8] based on nitrogen-plasma-source-based molecular beam epitaxy (MBE) eliminated the use of foreign metal, such as gold catalyst [9], which act as non-radiative recombination center and constitute material incompatibility with silicon foundry technology. The catalyst-free growth technology led to the subsequent demonstration of visible and white InGaN/GaN quantum-disk-in-nanowires light-emitting diodes (LEDs) on silicon [2, 10-14] and $\mathrm{SiO}_{2} / \mathrm{Si}$ [15]. Structurally, the nanostructures circumvented the thermal mismatch and lattice mismatch associated with heteroepitaxy integration, i.e. growth of $\mathrm{InGaN} / \mathrm{GaN}$ on the dissimilar substrates. Nanostructures thus prepared exhibited no threading dislocations, showing no quantum efficiency rollover, i.e. efficiency droop, low Auger recombination coefficient $\sim 10^{-33} \mathrm{~cm}^{6} \cdot \mathrm{s}^{-1}$ [16], and reduced quantum confined stark-effect in LEDs application. A plethora of promising devices was also demonstrated based on GaN nanowires, such as optically pumped single GaN nanowire laser based on photonic crystal microcavity [17], and vertically-integrated dielectric-mirror-clad polaritonlasers operating at room temperature [18-20].

*boon.ooi@kaust.edu.sa; $\underline{\text { http://photonics.kaust.edu.sa }}$

Gallium Nitride Materials and Devices IX, edited by Jen-Inn Chyi, Yasushi Nanishi, Hadis Morkoç, Joachim Piprek, Euijoon Yoon, Hiroshi Fujioka, Proc. of SPIE Vol. 8986, 898613 · @ 2014 SPIE CCC code: $0277-786 X / 14 / \$ 18 \cdot$ doi: $10.1117 / 12.2039627$ 
New nanostructures with different form factor (size and shape) and material composition will further enable growth of material structure with unique physical properties (thermal and lattice structure) for wavelength tuning and bandgap engineering of active region and thus enable creation of novel devices. In this investigation, we present the properties and the growth kinetics of defect-free and photoluminescence-efficient mushroom-like nanowires (MNW) in the form of $\sim 30 \mathrm{~nm}$ thick hexagonal-shaped InGaN-nanodisk-on-GaN-nanowire grown using Molecular Beam Epitaxy (MBE).

\section{EXPERIMENTAL DETAILS}

The InGaN-on-GaN-nanowire sample was grown on silicon (111) substrate using a molecular beam epitaxy (MBE) system having ultra-high purity gallium and indium sources, in conjunction with nitrogen-plasma source and conditions similar to references $[21,22]$. The native oxide was removed from the substrate surface before epitaxy by the standard technique, which involves a rinse in $\mathrm{HF}-\mathrm{H}_{2} \mathrm{O}$ solution and heat treatment in the growth chamber at $900{ }^{\circ} \mathrm{C}$ for $30 \mathrm{~min}$ at the vacuum level of $1 \times 10^{-9}$ Torr. The substrate temperature was lowered to $800{ }^{\circ} \mathrm{C}$ and a few monolayers of Ga were deposited with a gallium flux of $1.5 \times 10^{-7}$ Torr in the absence of nitrogen flux. The catalyst-free growth was initiated with binary GaN grown to a nominal thickness of $150 \mathrm{~nm} \mathrm{GaN}$ at $800{ }^{\circ} \mathrm{C}$ under nitrogen-rich conditions. A growth interruption was introduced whereby the growth temperature was reduced to $<600{ }^{\circ} \mathrm{C}$, and the indium shutter was opened to continue with the InGaN nanowires growth to a nominal thickness of $500 \mathrm{~nm}$. The optical properties and morphology of the nanowires were studied using scanning electron microscopy (SEM), and confocal microscopy (CFM) as well as micro-photoluminescence (PL) spectroscopy, respectively. Both CFM and PL are equipped with CCD-based spectrometer.

\section{RESULTS AND DISCUSSION}

On the same sample we observe two types of nanostructures, i.e. the InGaN-mushroom-cap on GaN nanowires, henceforth, the mushroom-like nanowires (MNW) growth in the form of $\sim 30 \mathrm{~nm}$ thick hexagonal-shaped InGaNnanodisk on GaN nanowire, coexisting with the conventional rod-like InGaN-on-GaN nanowires (RNW), see Fig.1(a) and (b) respectively. It is noted that the MNWs in Fig.1(a) were observed at the round spots with diameter of $\sim 30-40$ $\mu \mathrm{m}$, which is the grey-color round spot in the optical microscope picture in Fig.1(c), while the region outside the round spots were overgrown with RNWs (purple-color region outside the round spot). The round grey color spots were sparsely populated and more of such feature were found close to the MBE substrate holder retainer pins, which facilitated heat dissipation and therefore local temperature is lower than the central area region. At the center of the round spot, in addition to the MNWs, chunky materials with irregular shapes were found indicating severe distortion of the crystal structure, likely due to the formation of polycrystalline materials with dislocations formation.
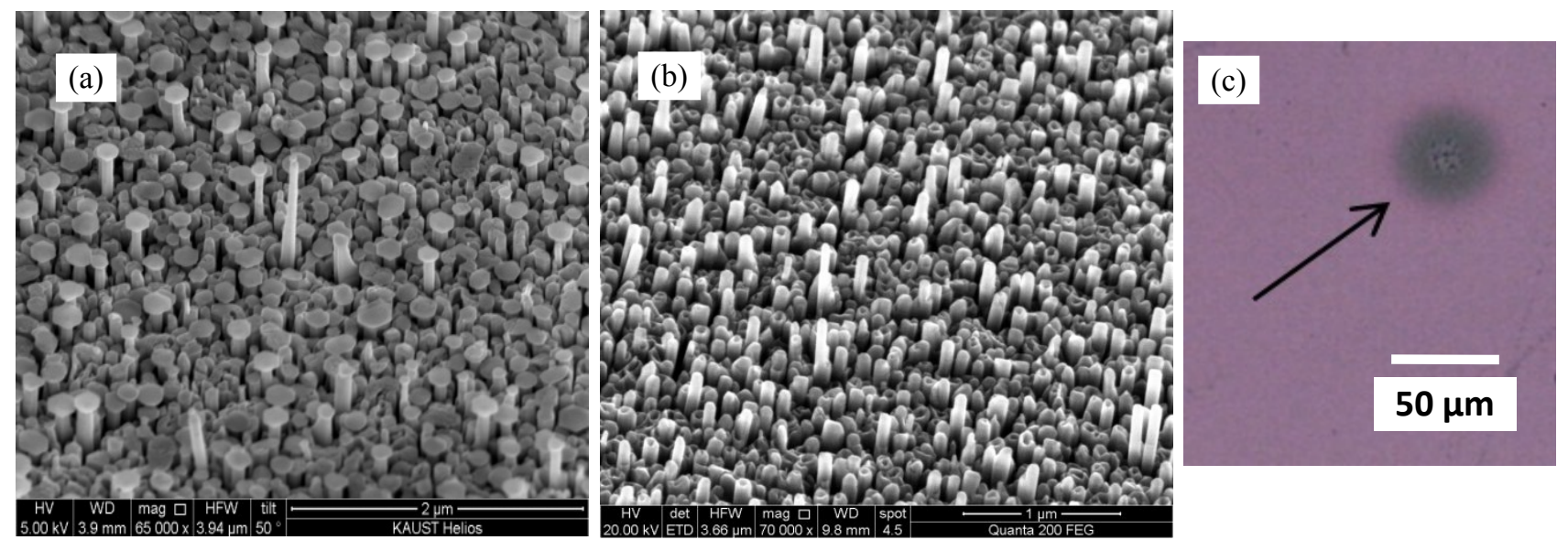

Fig.1 Scanning electron microscopy (SEM) images of (a) InGaN-mushroom-cap on GaN nanowires (MNW), and (b) InGaN-on-GaN nanowires. The microscope picture in (c) shows the grey-color region (as indicated by a pointed arrow) where MNWs was found, and the purple-color region where the conventional RNWs were observed.

The spontaneous emission was collected using from the MNWs and RNWs regions using PL spectroscopy with 473nm laser excitation having a laser beam spot of between 1-2 $\mu \mathrm{m}$. As shown in Fig.2(a), a broad PL spectrum with 3 main peaks covering the entire visible wavelength range was measured for the MNWs spot; while a narrower PL spectrum 
with peak emission at 490nm was observed for the RNW region (see Fig.2(b)). With arbitrary Gaussian fittings, five peaks were obtained with three main peaks located at $500 \mathrm{~nm}, 570 \mathrm{~nm}$ and $710 \mathrm{~nm}$, as well as two other peaks at $610 \mathrm{~nm}$ and $640 \mathrm{~nm}$. The peaks were originated from MNWs with varying indium compositions.

In order to ascertain the spatial distribution of the PL peaks and therefore the distribution of indium composition, the area shown in Fig.1(c) was characterized using confocal microscopy (CFM) with 458nm laser excitation. This is done by measuring the spontaneous emission map with detection wavelength swept at $\sim 10 \mathrm{~nm}$ steps. With increasing detection wavelength, the spatial spontaneous emission map as shown in Fig.3(a)-(c), respectively, changes from pixelated emission from the whole area, to having only an emission ring, and then a round emission spot. This corresponds to the PL emission with increasing indium composition; starting from emission mainly from the RNW, and then the emission from the MNWs at 540nm, and the emission from MNWs at $590 \mathrm{~nm}$. The spatial maps of MNWs with varying indium composition indicated that the indium composition increases from the circumference of the round spot towards the center. It is hence clear that local accumulation of indium occurred during growth, and hence facilitated the formation of these unique mushroom-like nanowires (MNWs).
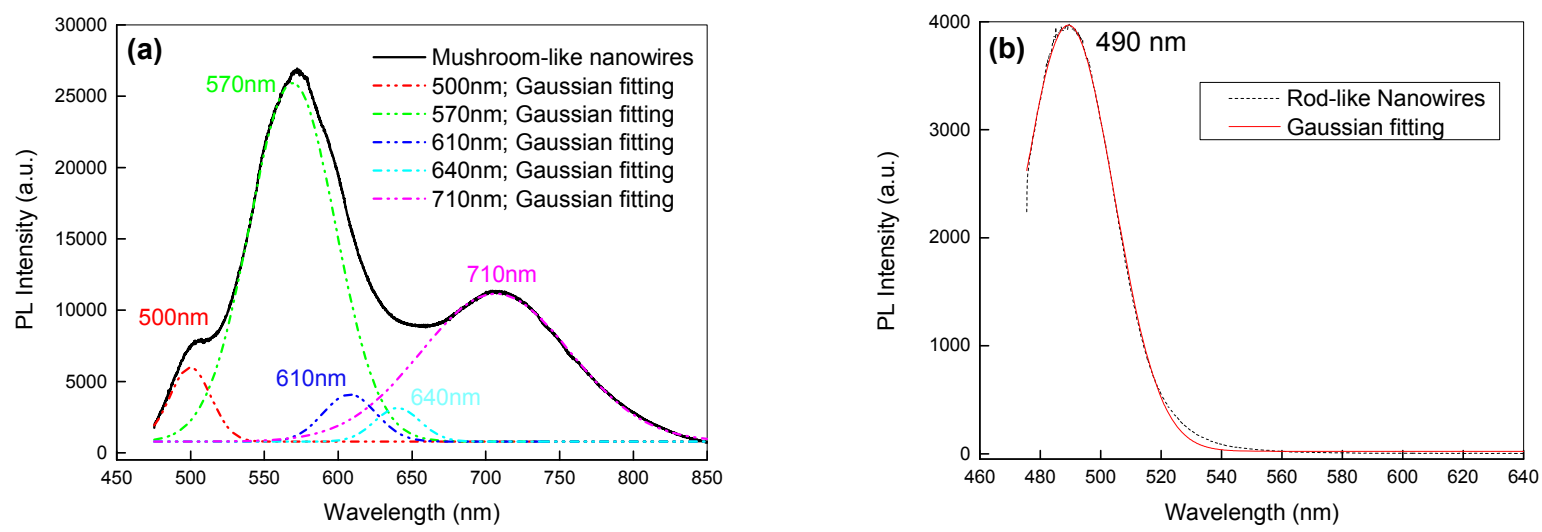

Fig.2 The photoluminescence spectra (black curve) of the sample at the region having: (a) the InGaN-mushroom-cap on $\mathrm{GaN}$ nanowires (MNWs), and (b) the conventional rod-like nanowires (RNWs). The spectrum for MNWs was fitted with five arbitrary Gaussian curves with peak wavelengths located at $500 \mathrm{~nm}, 570 \mathrm{~nm}, 610 \mathrm{~nm}, 640 \mathrm{~nm}$, and $710 \mathrm{~nm}$, while the spectrum for the RNWs was fitted with single Gaussian curve. Both spectra were measured using $325 \mathrm{~nm}$ and $473 \mathrm{~nm}$ laser excitation, respectively.
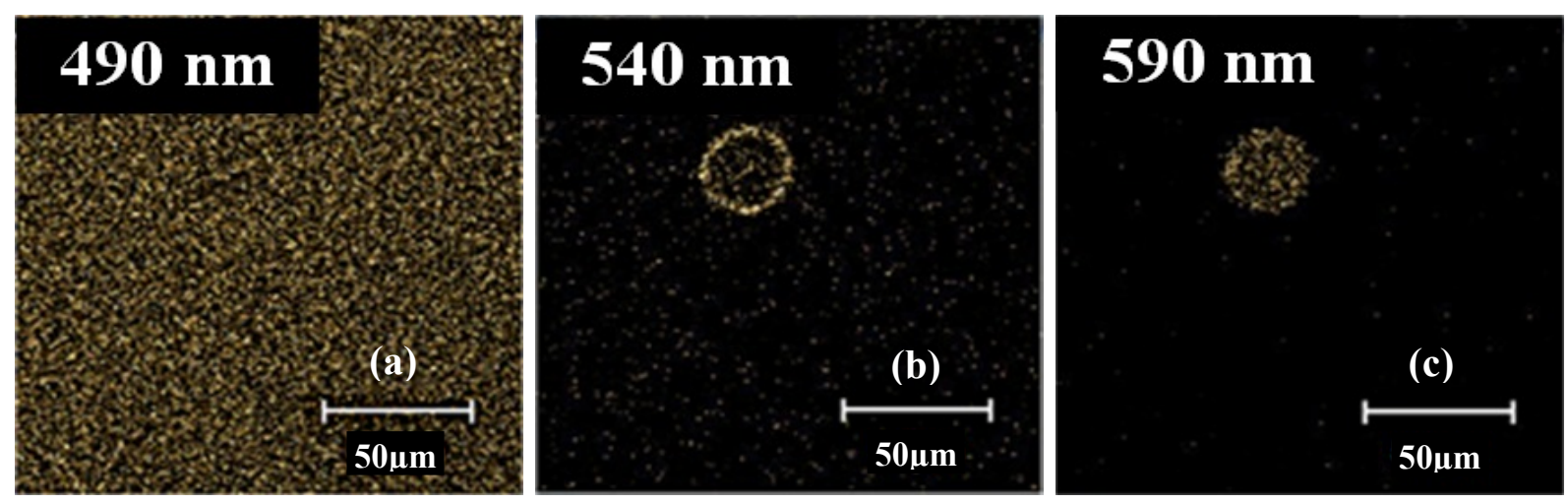

Fig.3 The confocal microscope (CFM) based photoluminescence map of InGaN nanowire at detection wavelength of (a) 490nm, (b) $540 \mathrm{~nm}$, and (c) $590 \mathrm{~nm}$. Spatially uniform emission from the rod-like InGaN NW was detected at $490 \mathrm{~nm}$, while the $25 \mu \mathrm{m}$ diameter region showed a clear transition from a ring emission peaking at $540 \mathrm{~nm}$ (emission from crystalline InGaN-nano-disk on GaN-NW, i.e. mushroom-like NW), to a uniform circular emission towards $590 \mathrm{~nm}$ (and beyond) detection wavelength (a mixed of crystalline/polycrystalline/amorphous (In)GaN). 
The spontaneous growth of GaN nanowires proceeded without the use of catalyst, and is governed by the kinetic processes $[8,22]$. As shown in the schematic of Fig.4(a), during the high temperature growth $\left(800{ }^{\circ} \mathrm{C}\right)$ of the $\mathrm{GaN}$ nanowires, the impinging gallium flux gets adsorbed (physical attachment) on the silicon (111) substrate. These adatoms either gets desorbed or continues to migrate before they eventually nucleate the initial basal GaN nanowires after combining with nitrogen species. Since the gallium ad-atoms have enough kinetic energy, they will diffuse up the length of the nanowires and nucleate on top of the nanowires as well as diffuse away from the basal GaN nanowires at high growth temperature (long ad-atoms diffusion length). The preferential growth along [0001], or c-direction under nitrogen-rich conditions and high temperature led to rod-like GaN nanowire growth. In addition, the direct impingement of gallium flux on the nanowire also contributed to GaN nucleation at the top. Such growth mechanism is common for the RNW and MNW during GaN growth, and hence rod-like structures were formed for both types of nanowires.

Although the nanowires growth were conducted using group- $\mathrm{V}$ rich conditions, the growth interruption time between 800 to $<600{ }^{\circ} \mathrm{C}$ in preparation for InGaN growth provided time and the required activation energy for the dissociation of $\mathrm{GaN}$; desorption of gallium and dissociation of $\mathrm{GaN}$ at high temperature limit, and local accumulation of gallium during the lower temperature limit (close to $<600{ }^{\circ} \mathrm{C}$ ) as mentioned. We now turn to explaining the kinetics behind the local formation of MNW. While the rod-like InGaN growth proceeded in a manner similar to that of rod-like GaN described in the above (see steps 1-4 in Fig.4(a)), the InGaN mushroom-disk growth is postulated to proceed through steps 1-2 as shown in Fig.4(b) due to a short migration length of ad-atoms. It is postulated to arise from the highly temperature sensitive growth kinetics during plasma-assisted $\mathrm{MBE}$ of $\mathrm{InGaN}$ during the temperature transition, i.e. when the substrate temperature was reduced from $800{ }^{\circ} \mathrm{C}$ ( $\mathrm{GaN}$ growth) to $<600{ }^{\circ} \mathrm{C}$ (InGaN growth), during which sparsely populated group-III metal-droplet formation prevails and further accumulated incoming indium ad-atoms due to higher cohesive bond between metallic molecules and lower substrate temperature $<600{ }^{\circ} \mathrm{C}$. This is typically found during the growth of nitride epitaxy at intermediate growth regime or III/V ratio $\sim 1$, (see microscope picture in Fig.5), where the droplets in the size of tens of micrometer were routinely found. Similar mechanism led to the droplet formation.

(a) Rod-like nanowire growth

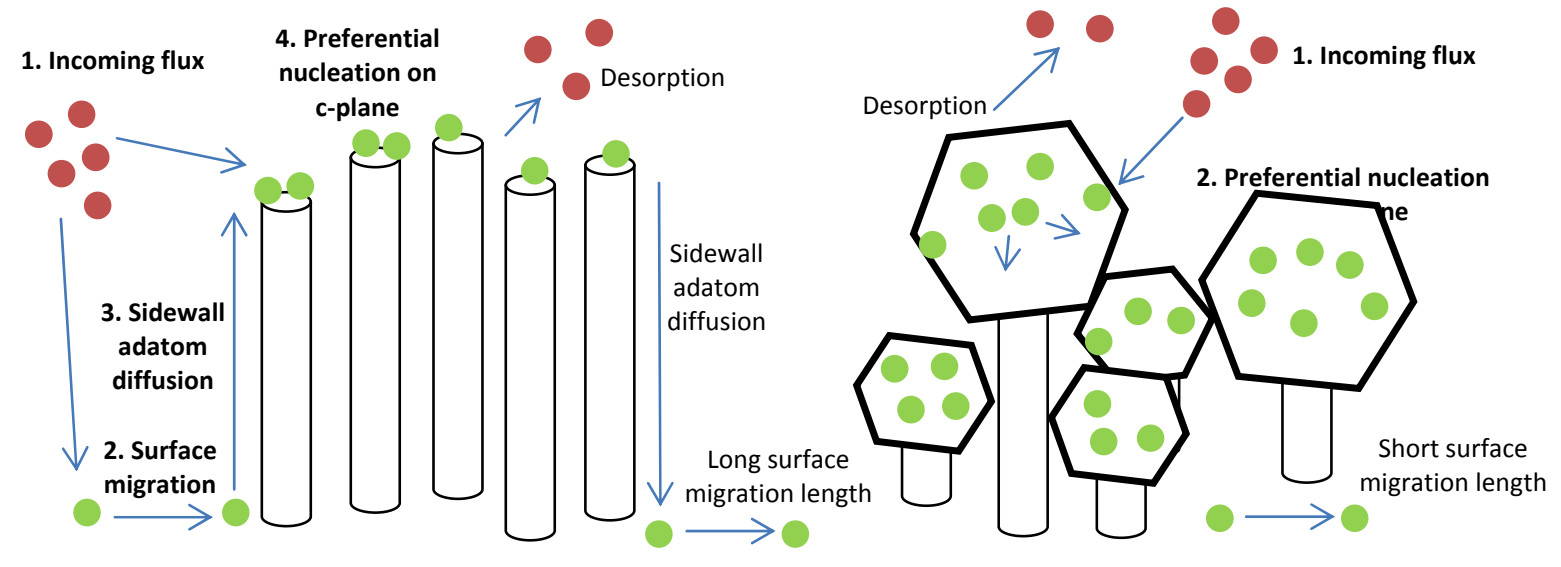

Fig.4 Schematic diagram showing growth kinetics in: (a) InGaN-on-GaN rod-like nanowire, and (b) InGaN-disk-on-GaNnanowire or mushroom-like nanowire growth on silicon (111) substrate. The green balls represent the group-III ad-atoms on substrate surface or nanowire surface, while the red balls represent incoming or outgoing (desorption) group-III fluxes. Incoming Group-V flux is not shown. During the top InGaN growth, nitrogen-rich and local metal-rich conditions resulted in the growth of rod-structure through steps 1-4, and hexagonal-disk-structure through step 1-2 in (a) and (b), respectively. 


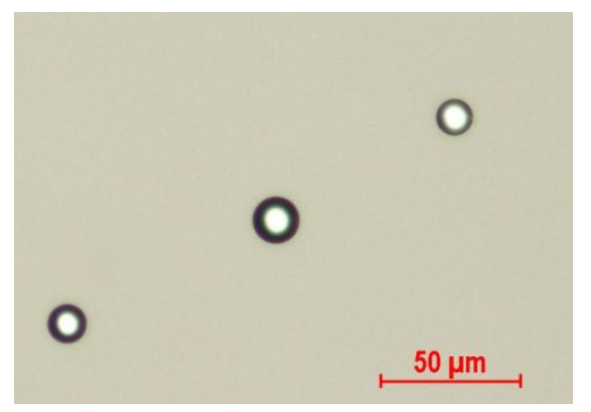

Fig.5 Microscope picture showing the typical formation of group-III droplets (the 3 circular spots) on GaN surface during growth of nitride epitaxy at temperature $\sim 600{ }^{\circ} \mathrm{C}$ at intermediate growth regime, i.e. III/V ratio slightly higher than 1 .

The energy dispersive X-ray spectroscopy (EDXS, not shown here) showed the basal rod of the MNWs consisted of only $\mathrm{GaN}$ while the hexagonal cap consisted of InGaN. This evidence allows us to further explain the formation of the hexagonal InGaN disk with the six facets corresponded to the m-planes. The presence of local metal-rich region and low InGaN growth temperature led to the two-dimensional (2D) growth of InGaN disk and preferential nucleation at the $\mathrm{m}$ planes rather than the nucleation at the c-plane. The preferentially nucleation at the m-plane facets, instead of c-plane is also due to strain-induced lateral crystal expansion growth in the presence of more metallic species (see Fig.4(b)). The understanding of the growth mechanism suggested a potential way of pendeo-epitaxial growth [23] based on the coalescence of well-oriented InGaN hexagonal disk on hexagonally patterned substrate.

\section{CONCLUSIONS}

We reported on the optical properties and concurrent formation growth kinetics of the rod-like nanowires (RNWs) and mushroom-like nanowires (MNWs). It is noted that the simultaneous emission from the NRW and MNW forms white light and therefore is attractive for solid state lighting. The PL emission from the MNWs was spatially resolved using CFM, and provided the evidence of local indium accumulation during growth. The local metal-rich condition and low temperature growth of InGaN led to the growth of hexagonal cap disk and the MNW. The understanding of the growth mechanism suggested a potential way of pendeo-epitaxial growth for $\mathrm{InGaN}$ template substrate formation based on the coalescence of the well-oriented and equal height InGaN hexagonal disk, though the orientation control is potentially a great research challenge.

\section{ACKNOWLEDGEMENTS}

T. K. Ng gratefully acknowledges the funding support from KAUST Competitive Research Grant.

\section{REFERENCES}

[1] Calarco, R., Meijers, R. J., Debnath, R. K., Stoica, T., Sutter, E., and Lüth, H., "Nucleation and Growth of GaN Nanowires on Si(111) Performed by Molecular Beam Epitaxy," Nano Letters 7, 2248-2251 (2007).

[2] Li S., and Waag, A., "GaN based nanorods for solid state lighting," Journal of Applied Physics 111, 071101 (2012).

[3] Murotani, H., Yamada, Y., Tabata, T., Honda, Y., Yamaguchi, M., and Amano, H., "Effects of exciton localization on internal quantum efficiency of InGaN nanowires," Journal of Applied Physics 114, 153506 (2013).

[4] Goodman, K. D., Protasenko, V. V., Verma, J., Kosel, T. H., Xing, H. G., and Jena, D., "Green luminescence of InGaN nanowires grown on silicon substrates by molecular beam epitaxy," Journal of Applied Physics 109, $084336(2011)$.

[5] Yoshizawa, M., Kikuchi, A., Mori, M., Fujita, N., and Kishino K., "Growth of Self-Organized GaN Nanostructures on $\mathrm{Al}_{2} \mathrm{O}_{3}(0001)$ by RF-Radical Source Molecular Beam Epitaxy," Japanese Journal of Applied Physics 36, L459L462 (1997).

[6] Sanchez-Garcia, M. A., Calleja, E., Monroy, E., Sanchez, F. J., Calle, F., Muñoz, E., et al., "The effect of the III/V ratio and substrate temperature on the morphology and properties of $\mathrm{GaN}$ - and AlN-layers grown by molecular beam epitaxy on Si(1 11 1)," Journal of Crystal Growth 183, 23-30 (1998). 
[7] Bertness, K. A., Sanford, N. A., Barker, J. M., Schlager, J. B., Roshko, A., Davydov, A. V., et al., "Catalyst-free growth of GaN nanowires," Journal of Electronic Materials 35, 576-580 (2006).

[8] Fernández-Garrido, S., Kong, X., Gotschke, T., Calarco, R., Geelhaar, L., Trampert, A., et al., "Spontaneous Nucleation and Growth of GaN Nanowires: The Fundamental Role of Crystal Polarity," Nano Letters 12, 61196125 (2012).

[9] Ma, Z., McDowell, D., Panaitescu, E., Davydov, A. V., Upmanyu, M., and Menon, L.,"Vapor-liquid-solid growth of serrated GaN nanowires: shape selection driven by kinetic frustration," Journal of Materials Chemistry C 1, 7294-7302 (2013).

[10] Jahangir, S., Mandl, M., Strassburg, M., and Bhattacharya, P., "Molecular beam epitaxial growth and optical properties of red-emitting (lambda $=650 \mathrm{~nm}$ ) $\mathrm{InGaN} / \mathrm{GaN}$ disks-in-nanowires on silicon," Applied Physics Letters 102, 071101 (2013).

[11] Guo, W., Banerjee, A., Bhattacharya, P., and Ooi, B. S., "InGaN/GaN disk-in-nanowire white light emitting diodes on (001) silicon," Applied Physics Letters 98, 193102 (2011).

[12] Guo, W., Zhang, M., Banerjee, A. and Bhattacharya, P., "Catalyst-Free InGaN/GaN Nanowire Light Emitting Diodes Grown on (001) Silicon by Molecular Beam Epitaxy," Nano Letters 10, 3355-3359 (2010).

[13] Nguyen, H. P. T., Zhang, S., Cui, K., Han, X., Fathololoumi, S., Couillard, M., et al., "p-Type Modulation Doped InGaN/GaN Dot-in-a-Wire White-Light-Emitting Diodes Monolithically Grown on Si(111)," Nano Letters 11, 1919-1924 (2011).

[14] Lin, H.-W., Lu, Y.-J., Chen, H.-Y., Lee, H.-M., and Gwo, S., "InGaN/GaN nanorod array white light-emitting diode," Applied Physics Letters 97, 073101 (2010).

[15] Zhao, S., Kibria, M. G., Wang, Q., Nguyen, H. P. T., and Mi, Z., "Growth of large-scale vertically aligned GaN nanowires and their heterostructures with high uniformity on SiOx by catalyst-free molecular beam epitaxy," Nanoscale 5, 5283-5287 (2013).

[16] Guo, W., Zhang, M., Bhattacharya, P., and Heo, J., "Auger Recombination in III-Nitride Nanowires and Its Effect on Nanowire Light-Emitting Diode Characteristics," Nano Letters 11, 1434-1438 (2011).

[17] J. Heo, W. Guo, and P. Bhattacharya, "Monolithic single GaN nanowire laser with photonic crystal microcavity on silicon," Applied Physics Letters 98, 021110 (2011).

[18] Heo, J., Jahangir, S., Xiao, B., and Bhattacharya, P., "Room-Temperature Polariton Lasing from GaN Nanowire Array Clad by Dielectric Microcavity," Nano Letters 13, 2376-2380 (2013).

[19] Das, A., Heo, J., Jankowski, M., Guo, W., Zhang, L., Deng. H., et al., "Room Temperature Ultralow Threshold GaN Nanowire Polariton Laser," Physical Review Letters 107, 066405 (2011).

[20] Das, A., Heo, J., Jankowski, M., Guo, W., Zhang, L., Deng, H., et al., "Room Temperature Polariton Lasing from a Single GaN Nanowire Microcavity," IEEE Photonics Conference (IPC) 119-120 (2011).

[21] Zhang, M., Bhattacharya, P., and Guo, W.,"InGaN/GaN self-organized quantum dot green light emitting diodes with reduced efficiency droop," Applied Physics Letters 97, 011103 (2010).

[22] Dubrovskii, V. G., Sibirev, N. V., Harmand, J. C., and Glas, F., "Growth kinetics and crystal structure of semiconductor nanowires," Physical Review B 78, 235301 (2008).

[23] Zheleva, T. S., Smith, S. A., Thomson, D. B., Linthicum, K. J., Rajagopal, P. and Davis, R. F., "Pendeo-epitaxy: a new approach for lateral growth of gallium nitride films," J. Electron. Mater. 28, L5-L8 (1999). 